\title{
A characterization of two disproportionality and malapportionment indices: the Duncan and Duncan index and the Lijphart index
}

\author{
D. Bouyssou · T. Marchant • M. Pirlot
}

Received: date / Accepted: date

\begin{abstract}
Disproportionality indices aim at measuring to what extent the composition of a parliament differs from the distribution of the votes among parties. Malapportionment indices measure to what extent the number of parliament seats attached to each district difffers from the distribution of the population among districts. Since there exist many different such indices, some conditions have recently been proposed for assessing the merits of the various indices. In this paper, we propose a characterization of two disproportionality and malapportionment indices: the Duncan and Duncan index (also called Loosemore-Hanby) and the Lijphart index.
\end{abstract}

Keywords disproportionality index · malapportionment · proportional representation · Duncan and Duncan · Loosemore-Hanby · Lijphart

\section{Introduction}

Suppose 100 voters are to elect 10 representatives in a parliament. There are 3 political parties respectively receiving 25,43 and 32 votes. If we want to achieve proportional representation, the three parties should respectively have

Authors are listed alphabetically and have contributed equally.

D. Bouyssou

LAMSADE, CNRS, UMR 7243, Université Paris-Dauphine, PSL Research University, 75016

Paris, France

E-mail: bouyssou@lamsade.dauphine.fr

T. Marchant

Ghent University, Dunantlaan 1, 9000 Ghent, Belgium

E-mail: thierry.marchant@ugent.be

M. Pirlot

Université de Mons, rue de Houdain 9, 7000 Mons, Belgium

E-mail: marc.pirlot@umons.ac.be 
2.5 (i.e., $10 \times 25 / 100$ ), 4.3 and 3.2 representatives $^{1}$. This ideal representation is not feasible because it is not integer. Hence, one has to choose between one of the many different ways to obtain an integer number of representatives. Many allocation methods (for instance the Hare quota or the Droop quota) for choosing the right distribution of representatives have been proposed [2]. In order to compare these methods, or more precisely to compare the outcomes of these methods, political scientists have proposed several indices to measure the dissimilarity between the perfect proportional (but usually non-integer) distribution and a feasible one: the so-called disproportionality indices. The first one was the Rae index [17], soon followed by many others [10]. Around the year 2000, there were so many disproportionality indices at hand that political scientists realized that a systematic approach to the analysis of disproportionality indices was necessary. ${ }^{2}$ This led them to proposing some conditions or properties that disproportionality indices should ideally satisfy $[4,10,11,18]$, but no characterization of a disproportionality index has been proposed so far in political sciences ${ }^{3}$.

A related problem is the apportionment of seats among electoral districts. There also, some malapportionment indices have been proposed; they are formally equivalent to disproportionality indices and are also discussed by $[4,10,11,18]$.

Another related problem is that of comparing two probability distributions. For instance, [7] reviews more than 60 dissimilarity indices between two probability distributions. If we model a parliament by means of proportions of seats, then dissimilarity indices (between two probability distributions) and disproportionality indices (between a seat distribution and a vote distribution) are formally equivalent. But such a modelling would implicitly assume a homogeneity hypothesis (i.e. the size of a parliament does not matter; only proportions matter). We do not consider this homogeneity hypothesis as compelling and we therefore consider our problem as related but distinct from the measurement of dissimilarity between two probability distributions.

We will not propose and champion a new disproportionality index or support an existing one because we think that a single best universal disproportionality index does not exist. For instance, we probably do not need the same index if we want to measure malapportionment in a small or in a large country. Instead of supporting a specific index, we will provide a formal framework in which it is possible to analyze and compare various indices, using clear and explicit arguments. This is in line with the authors' previous work in decision theory [e.g. 5, 6].

\footnotetext{
1 In a recent paper, [16] argues that the concept of proportional representation is both vague and ambiguous. To make things clear, we consider one-option balloting and proportional allocation of seats.

$2[10,11]$ call this an axiomatic approach.

3 No disproportionality index has been characterized, yet several characterizations of allocation methods (i.e., techniques to allocate seats to parties in view of the electoral result) are available in the literature [2].
} 


\section{Definitions, notation and some indices}

In this section, we introduce the notation that will be needed. We also define several disproportionality indices.

\subsection{Notation and definitions}

The set $N=\{1,2, \ldots, n\}$, with $n \geq 2$, represents the set of parties or electoral districts. We will throughout consider that $N$ does not vary. We will consider that the distribution of votes among parties can adequately be described by a vector with rational components ${ }^{4}$. This distribution will be called the target distribution or just target. The set of non-negative rational numbers is denoted by $\mathbb{Q}_{+}$. The set of strictly positive (resp. non-negative) real numbers is denoted by $\mathbb{R}_{++}$(resp. $\mathbb{R}_{+}$). The set of natural numbers is denoted by $\mathbb{N}$ and $\mathbb{N}_{0}=$ $\mathbb{N} \backslash\{0\}$. The target distribution is $\pi=\left(\pi_{1}, \ldots, \pi_{n}\right)$, with $\pi_{i} \in \mathbb{Q}_{+}$for all $i \in N$ and $\sum_{i \in N} \pi_{i}=1$. In the context of proportional seat allocation, $\pi$ is the vector of the relative vote shares among the parties; in the context of apportionment, it is the vector of the relative population shares among the districts.

A parliament is described by the distribution of seats among parties. It is denoted by $x=\left(x_{1}, \ldots, x_{n}\right)$, with $x_{i} \in \mathbb{N}$ for all $i \in N$ and where $x_{i}$ represents the number of seats allocated to party or district $i$. The size of parliament $x$ is denoted by $s(x)=\sum_{i \in N} x_{i}$. The relative frequency distribution in a parliament $x$ is $x / s(x)=\left(x_{1} / s(x), \ldots, x_{n} / s(x)\right)$. The set of all parliaments is $X=\mathbb{N}^{N}$, i.e. the set of all mappings from $N$ to $\mathbb{N}$. The set $\Pi$ of all target distributions is a subset of $\mathbb{Q}_{+}^{N}$. Formally, $\Pi=\left\{\pi \in \mathbb{Q}_{+}^{N}: \sum_{i \in N} \pi_{i}=1\right\}$.

Definition 1 A disproportionality index $f$ is a mapping from $X \times \Pi$ to $\mathbb{R}_{+}$ satisfying $f(x, \pi)=0$ iff $x / s(x)=\pi$.

A disproportionality index measures how far from the target $\pi$ the actual seat distribution $x$ in the parliament is. It is equal to zero only when the seat distribution is perfectly proportional.

The reader may have the impression that comparisons of disproportionality indices across different elections are not possible in our setting because the set $N$ of parties is fixed while the set of parties in real elections is almost never the same. This is not completely correct. Indeed, we can consider some very large set $N$ (containing e.g. 100 parties) and, when we want to model a particular election involving a subset $M \subset N$ of parties, we just ensure that the parties in $N \backslash M$ have 0 votes and 0 seats. We will discuss this in detail in Section 4.

\subsection{Two disproportionality indices}

We begin with an additive index: $f_{\mathrm{DD}}(x, \pi)=\sum_{i \in N}\left|x_{i} / s(x)-\pi_{i}\right|$. For each party $i$, we compute the deviation $x_{i} / s(x)-\pi_{i}$ between the proportion in

\footnotetext{
4 We thus implicitly consider that the size of the set of voters does not matter.
} 
the parliament $\left(x_{i} / s(x)\right)$ and the target proportion $\left(\pi_{i}\right)$. We summarize the $n$ such deviations by means of their sum after taking absolute values in order to avoid compensation between positive and negative deviations. Index $f_{\mathrm{DD}}$ is the Duncan and Duncan [8] (also called Loosemore-Hanby [13]) index. ${ }^{5}$

A different index is $f_{\mathrm{L}}(x, \pi)=\max _{i \in N}\left|x_{i} / s(x)-\pi_{i}\right|$. Here, instead of using the sum as a summary, we focus on the largest absolute deviation. Index $f_{\mathrm{L}}$ (or Lijphart index) has been proposed in [12].

Indices $f_{\mathrm{DD}}$ and $f_{\mathrm{L}}$ correspond to $L^{p}$ distances (with $p=1$ and $\infty$ resp.) between the vectors $\pi$ and $x / s(x)$. Many more indices can be defined by just considering other values of $p$ or other distances. If we do not limit ourselves to distances, many other indices can be thought of. For instance, [18] discusses nineteen disproportionality indices.

\section{Characterizations}

As already mentioned in the introduction, we do not promote a particular index. For the same reason, we will not try to justify our properties on normative grounds. Properties need not be compelling: they should be simple properties, easy to understand and as elementary as possible. They allow us to break down an index (possibly defined by a complex formula) into a small number of elementary and simple blocks. And if one of the properties characterizing an index is easy to understand but the social planner does not find it adequate, then he has a good reason not to use that index. For a deep discussion about the utility of this kind of analysis, we refer to [14, pp.196-202]. See also [3, Chapter 1] in which various views on using characterizations are described and compared. Our view, in the context of disproportionality measurement, is close to that of [19], in the context of game theory and resource allocation.

In this paper, we characterize two simple and popular indices: $f_{\mathrm{DD}}$ and $f_{\mathrm{L}}$. These two characterizations should be considered as examples of what can be achieved and expected within the framework we propose.

\subsection{Characterization of index $f_{\mathrm{DD}}$}

\subsubsection{Properties}

Homogeneity. Suppose $n=2$ and party 1 has 400 seats in parliament $x$ with size $s(x)=600$ and 800 seats in parliament $y$ with $s(y)=1200$. In terms of proportions, $x$ and $y$ are identical and this suggests that the disproportionality index (given the same target $\pi$ ) should be equal in the two parliaments. The first property we discuss formalizes this idea: it imposes that the size of a

\footnotetext{
5 The Duncan and Duncan index is often presented as $1 / 2 \times \sum_{i \in N}\left|\pi_{i}-x_{i} / s(x)\right|$. The factor $1 / 2$ is convenient in applications because it scales the index between 0 and 1 . We purposefully drop this factor because it makes the index simpler. The difference between the two forms of the index is not more relevant than the difference between a length measurement in meters or in feet.
} 
parliament does not matter, in the sense that scaling up or down a parliament by a multiplicative factor does not change its index.

P 1 Homogeneity. For all $\lambda \in \mathbb{N}_{0}$ and all $x \in X, f(\lambda x, \pi)=f(x, \pi)$.

Let us show that index $f_{\mathrm{DD}}$ satisfies Homogeneity.

$f_{\mathrm{DD}}(\lambda x, \pi)=\sum_{i \in N}\left|\pi_{i}-\frac{\lambda x_{i}}{s(\lambda x)}\right|=\sum_{i \in N}\left|\pi_{i}-\frac{\lambda x_{i}}{\lambda s(x)}\right|=\sum_{i \in N}\left|\pi_{i}-\frac{x_{i}}{s(x)}\right|=f_{\mathrm{DD}}(x, \pi)$.

Although Homogeneity seems appealing, if we consider very small and very large parliaments, then it is perhaps no longer so compelling. Suppose $n=2$, $s(x)=6, s(y)=600$ and the target is fifty-fifty. Suppose party 1 has 4 seats in parliament $x$. Then 4 seats for party 1 with respect to a target of 3 seems a very small deviation, not really indicating a representativity problem. On the contrary, 400 seats for party 1 in parliament $y$ with respect to a target of 300 seems problematic, and it seems reasonable to have a higher disproportionality index for $y$ than for $x$, although they are identical in terms of proportions. Even worse: suppose party 1 has two seats in parliament $z$ with $s(z)=3$. In terms of proportions, $z$ is identical to $x$ and $y$. But a perfect proportionality is impossible in a parliament of size three with a fifty-fifty target. It would be unfair to associate the same disproportionality index to parliaments $z$ and $x$ or $y$. We move to another property.

Radial Linearity. Consider a target $\pi$ and two parliaments $x$ and $y$ with the same size. Then $s(x) \pi$ is a perfectly proportional parliament with the same size as $x$. Suppose that $x$ and $y$ lie on the same ray originating in $s(x) \pi$. Then, $(x+y) / 2$ lies between $x$ and $y$, on the same ray, and a very mild monotonicity requirement is that $f((x+y) / 2, \pi)$ lies between $f(x, \pi)$ and $f(y, \pi)$. This monotonicity is satisfied by $f_{\mathrm{DD}}$ and we could use it to characterize the ranking induced by $f_{\mathrm{DD}}$ (see Section 4.2 ), but we chose to characterize the index $f_{\mathrm{DD}}$. We thus need a stronger condition, fixing the exact position of $f((x+y) / 2, \pi)$ between $f(x, \pi)$ and $f(y, \pi)$. The simplest thing we can then do is to say that this position is exactly halfway and we can motivate this by a kind of principle of insufficient reason. This is formalized hereafter.

P 2 Radial Linearity. If $x$ and $y$ are two parliaments such that (a) $s(x)=$ $s(y),(x+y) / 2 \in X$ and $(b) x=\alpha s(x) \pi+(1-\alpha) y$ for some $\alpha \in[0,1]$, then

$$
f\left(\frac{x+y}{2}, \pi\right)=\frac{f(x, \pi)+f(y, \pi)}{2} .
$$

Radial Linearity is a necessary condition for $f_{\mathrm{DD}}$. Indeed, $f_{\mathrm{DD}}(x+y, \pi)$

$=\sum_{i \in N}\left|\pi_{i}-\frac{x_{i}+y_{i}}{2 s(x)}\right|=\frac{1}{2} \sum_{i \in N}\left|2 \pi_{i}-\frac{x_{i}+y_{i}}{s(x)}\right|=\frac{1}{2} \sum_{i \in N}\left|\pi_{i}-\frac{x_{i}}{s(x)}+\pi_{i}-\frac{y_{i}}{s(y)}\right|$. 
Since $x=\alpha s(x) \pi+(1-\alpha) y$, we know that $\left\{i \in N: x_{i} / s(x) \geq \pi_{i}\right\}=\{i \in N$ : $\left.y_{i} / s(y) \geq \pi_{i}\right\}$ and we have $f_{\mathrm{DD}}(x+y, \pi)$

$$
\begin{aligned}
& =\frac{1}{2} \sum_{i \in N}\left|\pi_{i}-\frac{x_{i}}{s(x)}+\pi_{i}-\frac{y_{i}}{s(y)}\right| \\
& =\frac{1}{2}\left(\sum_{i \in N}\left|\pi_{i}-\frac{x_{i}}{s(x)}\right|+\sum_{i \in N}\left|\pi_{i}-\frac{y_{i}}{s(y)}\right|\right)=\frac{f_{\mathrm{DD}}(x, \pi)+f_{\mathrm{DD}}(y, \pi)}{2} .
\end{aligned}
$$

Radial Linearity may seem a (too) strong condition and characterizing an index with an additive form such as $f_{\mathrm{DD}}$ with Radial Linearity may seem too obvious for being interesting. Actually, there are many non-additive indices satisfying Radial Linearity. Index $f_{\mathrm{L}}$ is such an index (see Section 3.2). The main effect of Radial Linearity is not to make things additive, but rather to force the index to vary linearly when a parliament deviates from perfect proportionality in a given direction. Notice also that, despite an additive structure, the index $f_{\chi^{2}}$ defined by [15] as

$$
f_{\chi^{2}}(x, \pi)=\sum_{i \in N}\left(\frac{\left(x_{i} / s(x)-\pi_{i}\right)^{2}}{\pi_{i}}\right)
$$

does not satisfy Radial Linearity.

Substitution. Let $\mathbf{1}^{i}$ be an element of $X$ such that $\mathbf{1}_{k}^{i}=0$ for all $k \in N \backslash\{i\}$ and $\mathbf{1}_{i}^{i}=1$. For example, $\mathbf{1}^{2}=(0,1,0, \ldots, 0)$. If we add $\mathbf{1}^{i}$ to a parliament, we obtain a new parliament where an extra seat has been allocated to party $i$.

Suppose $n=3$, the target $\pi$ is $(1 / 3,1 / 3,1 / 3)$ and consider the parliament $x=(10,40,50)$. Parties 2 and 3 are overrepresented. We move a seat from party 3 to party 2 , yielding $y=(10,41,49)$. Parties 2 and 3 are still overrepresented. It makes sense to consider that we did not improve the situation: nothing changed for the underrepresented parties; they did not gain a single seat. We may therefore request that $f(x, \pi)=f(y, \pi)$. Put differently, we can move a seat in a parliament from a party to another, without changing the index value, as long as both parties are in excess (or both in deficit) before and after the change.

P 3 Substitution. Suppose $\left[\left(x_{i}-1\right) / s(x) \geq \pi_{i}\right.$ and $\left.x_{j} / s(x) \geq \pi_{j}\right]$ or $[0<$ $x_{i} / s(x) \leq \pi_{i}$ and $\left.\left(x_{j}+1\right) / s(x) \leq \pi_{j}\right]$. Then $f(x, \pi)=f\left(x-\mathbf{1}^{i}+\mathbf{1}^{j}, \pi\right)$.

Notice that this condition is vacuous when $n=2$. Substitution is satisfied by $f_{\mathrm{DD}}$. We only show it for the first part of the condition (corresponding to the case of parties in excess).

$$
f_{\mathrm{DD}}\left(x-\mathbf{1}^{i}+\mathbf{1}^{j}, \pi\right)=\left|\frac{x_{i}-1}{s(x)}-\pi_{i}\right|+\left|\frac{x_{j}+1}{s(x)}-\pi_{j}\right|+\sum_{k \in N \backslash\{i, j\}}\left|\frac{x_{k}}{s(x)}-\pi_{k}\right| .
$$


The dimensions in excess in $x$ and $x-\mathbf{1}^{i}+\mathbf{1}^{j}$ are the same. The right-hand side of the above equation can therefore be rewritten as

$$
\left|\frac{x_{i}}{s(x)}-\pi_{i}\right|+\left|\frac{x_{j}}{s(x)}-\pi_{j}\right|+\sum_{k \in N \backslash\{i, j\}}\left|\frac{x_{k}}{s(x)}-\pi_{k}\right|=f_{\mathrm{DD}}(x, \pi) .
$$

Invariance. We need an additional condition to characterize $f_{\mathrm{DD}}$ when $\pi$ is not fixed. It says that, if a parliament is perfectly proportional and if we move a seat from a party to another (thus moving away from the target), then the value of the index is the same, whatever the target is.

$\mathbf{P} 4$ Invariance. For any $i, j \in N$ and any $\lambda \in \mathbb{N}_{0}$, if $\lambda \pi, \lambda \pi-\mathbf{1}^{i}+\mathbf{1}^{j}, \lambda \pi^{\prime}, \lambda \pi^{\prime}-$ $\mathbf{1}^{i}+\mathbf{1}^{j} \in X$ then $f\left(\lambda \pi-\mathbf{1}^{i}+\mathbf{1}^{j}, \pi\right)=f\left(\lambda \pi^{\prime}-\mathbf{1}^{i}+\mathbf{1}^{j}, \pi^{\prime}\right)$.

Index $f_{\mathrm{DD}}$ satisfies Invariance. Indeed,

$$
f_{\mathrm{DD}}\left(\lambda \pi-\mathbf{1}^{i}+\mathbf{1}^{j}, \pi\right)=\left|\frac{\lambda \pi_{i}-1}{\lambda}-\pi_{i}\right|+\left|\frac{\lambda \pi_{j}+1}{\lambda}-\pi_{j}\right|=\frac{2}{\lambda} .
$$

In the same way, $f_{\mathrm{DD}}\left(\lambda \pi^{\prime}-\mathbf{1}^{i}+\mathbf{1}^{j}, \pi^{\prime}\right)=2 / \lambda$. This proves that $f_{\mathrm{DD}}(\lambda \pi-$ $\left.\mathbf{1}^{i}+\mathbf{1}^{j}, \pi\right)=f_{\mathrm{DD}}\left(\lambda \pi^{\prime}-\mathbf{1}^{i}+\mathbf{1}^{j}, \pi^{\prime}\right)$.

Invariance is a kind of symmetry condition. It is, to some extent, a desirable property, but it comes at a cost. Suppose indeed $\pi=(1 / 3,1 / 3,1 / 3), \pi^{\prime}=$ $(1 / 300,149 / 300,150 / 300), x=(99,101,100), y=(0,150,150)$ and consider the following situations: (a) parliament $x$ combined with target $\pi$ and (b) parliament $y$ combined with $\pi^{\prime}$. In both situations, party one has one seat less than what it deserves. In situation (a), the relative deviation for party 1 is $1 / 100$ (it deserves 100 seats); in situation (b), the relative deviation for party 1 is $1 / 1$. One could therefore argue that $f(x, \pi)$ should be smaller than $f\left(y, \pi^{\prime}\right)$. Yet Invariance (with $\lambda=300, i=1$ and $j=2$ ) implies $f(x, \pi)=f\left(y, \pi^{\prime}\right)$.

\subsubsection{Results}

We can now state a first characterization theorem ${ }^{6}$.

Theorem 1 A disproportionality index $f$ satisfies Homogeneity, Radial Linearity and Substitution iff, for all $\pi \in \Pi$, there exists $\gamma_{\pi} \in \mathbb{R}_{++}$such that

$$
f(x, \pi)=\gamma_{\pi} \sum_{i \in N}\left|\frac{x_{i}}{s(x)}-\pi_{i}\right|=\gamma_{\pi} f_{D D}(x, \pi) .
$$

If Invariance is added to the list of necessary and sufficient conditions, then $\gamma_{\pi}=\gamma_{\pi^{\prime}}$ for all $\pi, \pi^{\prime} \in \Pi$. The properties are logically independent, but Substitution can be omitted when $n=2$.

\footnotetext{
6 A characterization of the $L^{1}$ distance (obviously linked to index $f_{\mathrm{DD}}$ ) already exists [9]. It is not completely relevant to our problem because the authors consider it as a distance between two points in $\mathbb{R}^{n}$ while we are interested in the 'distance' between a point in $\mathbb{N}^{N}$ and a point in $\mathbb{Q}^{N}$ with the constraint that the sum of the coordinates be 1.
} 
The first part of this result (without Invariance) is useful when $\pi$ is not allowed to vary. The second part allows us to make comparisons of indices across parliaments in different countries or across time, where $\pi$ is not constant.

The idea of the proof ${ }^{7}$ is as follows. We first assume $\pi$ is fixed and we show that $f$ depends only on the relative distribution $x / s(x)$, thanks to Homogeneity. Any parliament can then be described by its position $(x / s(x))$ in the simplex $\Pi$. We then partition the set $\Pi$ into several domains. Each domain is characterized by a set of parties in excess (i.e., with a proportion $x_{i} / s(x)$ larger than $\pi_{i}$ ). Using Radial Linearity, we show that $f$ varies linearly on each line segment in $\Pi$ having $\pi$ as an endpoint. Notice that such a line segment always belongs to a single domain. We then show that, within a domain, the set of all points with equal value of the index is a hyperplane, by Substitution. It follows that $f$ is an affine function in each domain and it can therefore be represented by (1).

\subsection{Characterization of index $f_{\mathrm{L}}$}

\subsubsection{Properties}

It is easy to check that $f_{\mathrm{L}}$ does not satisfy Substitution. Does this imply that $f_{\mathrm{L}}$ is a bad index? We do not think so, because it is not that obvious that Substitution is a compelling condition in all contexts, as illustrated in the following example. Suppose $\pi=(1 / 4,1 / 4,1 / 4,1 / 4)$; then, according to Substitution, $x=(0,20,25,55)$ and $y=(0,20,40,40)$ must have the same disproportionality. Yet people may have a different opinion and reason as follows: parliaments $x$ and $y$ do an equally bad job with respect to parties 1 and 2 , but $x$ does also discriminate between parties 3 and 4 , while $y$ does not. So, one could argue that the disproportionality index of $y$ should be lower than that of $x$. In such a case, Substitution could be questioned. We will therefore propose a slightly different condition, after introducing a new piece of notation.

For every $i \in N, \pi$ being fixed, define $P(i)=\left\{x \in X: \frac{x_{i}}{s(x)}-\pi_{i} \geq\right.$ $\left.\left|\frac{x_{j}}{s(x)}-\pi_{j}\right| \forall j \in N\right\}$. In words, $P(i)$ is the set of all parliaments such that party $i$ is in excess and has the maximal deviation in absolute value. Similarly, $M(i)=\left\{x \in X: \pi_{i}-\frac{x_{i}}{s(x)} \geq\left|\frac{x_{j}}{s(x)}-\pi_{j}\right| \forall j \in N\right\}$. The set $M(i)$ interprets similarly. For every $i, P(i)$ and $M(i)$ cannot be both empty.

Suppose $n=3$, the target $\pi$ is $(1 / 3,1 / 3,1 / 3)$ and consider the parliament $x=(30,30,40)$. The largest deviation w.r.t. the target occurs for party 3 . We move a seat from party 1 to party 2 , yielding $y=(29,31,40)$. The largest deviation w.r.t. the target still occurs for party 3 . It makes sense to consider that we did not improve the situation: nothing changed for the party with the maximal deviation; it did not gain or loose a single seat. We may therefore request that $f(x, \pi)=f(y, \pi)$. Put differently, we can move a seat in a par-

\footnotetext{
7 All proofs are deferred to Section 5
} 
liament from a party to another, without changing the index value, as long as the parties with maximal deviations remain so after the change. Formally,

$\mathbf{P} 5$ Substitution*. Suppose that $x$ and $x-\mathbf{1}^{i}+\mathbf{1}^{j} \in P(k)$ or $x$ and $x-\mathbf{1}^{i}+\mathbf{1}^{j} \in$ $M(k)$, with $k \notin\{i, j\}$. Then $f(x, \pi)=f\left(x-\mathbf{1}^{i}+\mathbf{1}^{j}, \pi\right)$.

Notice that this condition is vacuous when $n=2$. Substitution* is satisfied by $f_{\mathrm{L}}$. We show it only for the first part of the condition, the other part being similar. Since $x$ and $x-\mathbf{1}^{i}+\mathbf{1}^{j} \in P(k)$, with $k \notin\{i, j\}$, we have

$$
f_{\mathrm{L}}\left(x-\mathbf{1}^{i}+\mathbf{1}^{j}, \pi\right)=\left|\frac{x_{k}}{s(x)}-\pi_{k}\right|=f_{\mathrm{L}}(x, \pi) .
$$

\subsubsection{Results}

Proposition 1 (Particular case). If $n=2$ or 3, then indices $f_{D D}$ and $f_{L}$ are identical, up to a multiplicative constant. More precisely, $f_{L}=f_{D D} / 2$.

Proposition 1 no longer holds when $n \geq 4$. Indeed, let $x=(30,35,20,15)$, $y=(32,37,19,12)$ and $\pi=(1 / 4,1 / 4,1 / 4,1 / 4)$. Then $f_{\mathrm{L}}(x, \pi)=0.1$ and $f_{\mathrm{L}}(y, \pi)=0.13$ while $f_{\mathrm{DD}}(x, \pi)=0.3$ and $f_{\mathrm{DD}}(y, \pi)=0.38$. So, there is no $\lambda$ such that $f_{\mathrm{L}}=\lambda f_{\mathrm{DD}}$. We now turn to the general case.

Theorem 2 A disproportionality index $f$ satisfies Homogeneity, Radial Linearity and Substitution ${ }^{*}$ iff, $\forall \pi \in \Pi, \exists \gamma_{\pi} \in \mathbb{R}_{++}$s.t. $f(x, \pi)=\gamma_{\pi} \max _{i \in N}\left|\frac{x_{i}}{s(x)}-\pi_{i}\right|=$ $\gamma_{\pi} f_{L}(x, \pi)$. If Invariance is added to the list of necessary and sufficient conditions, then $\gamma_{\pi}=\gamma_{\pi^{\prime}}$ for all $\pi, \pi^{\prime} \in \Pi$. The properties are logically independent, but Substitution * can be omitted when $n=2$.

\section{Discussion}

We have shown that $f_{\mathrm{L}}$ and $f_{\mathrm{DD}}$ both satisfy Homogeneity, Radial Linearity and Invariance, while they differ only by the kind of substitution they satisfy.

\subsection{Properties for the measurement of disproportionality in the literature}

We mentioned in Section 1 the existence of some papers presenting properties for indices of disproportionality. In this discussion, we will focus on the eight properties presented in [11] because they largely overlap with properties in other papers and they are more precisely stated than in the other papers (notice though that they are stated for a proportionality index and not a disproportionality index, but it is easy to adapt them). For space reasons, we will not discuss the eight properties individually. Let us just make two remarks.

The eight properties are not strong enough to obtain a characterization. Indeed, the cosine measure discussed in [11] satisfies all eight properties, but the square or the square root of the cosine measure also satisfies all eight 
properties. So, we definitely need to define some new ones in order to characterize some index. But, of course, we use some of the properties proposed in [11] (their Scale Invariance is our Homogeneity) and some of our new properties, taken individually or jointly, imply or are implied by some properties in [11]. For instance, their Dalton's principle of transfers is implied by our Radial Linearity.

Second, we find some of the properties used in [11] irrelevant for characterizing an index. In particular, that the maximal value of the index should be 1. A maximal value equal to 100 (or any positive number) would be equally fine. It is just a matter of convenience, without any substantial consequence.

\subsection{Index vs ranking}

There are cases where we need a ranking of the parliaments and not an index. Since many indices induce the same ranking ${ }^{8}$, we need stronger properties for characterizing an index than for characterizing a ranking. In particular, the ranking induced by $f_{\mathrm{DD}}$ (resp. $f_{\mathrm{L}}$ ) is characterized by Homogeneity, Substitution (resp. Substitution*), Invariance (all restated in terms of rankings) and a monotonicity condition much weaker than Radial Linearity.

\subsection{Comparisons across elections}

Our setting assumes that the set $N$ of parties does not vary. Yet, if we want to compare the disproportionality of two parliaments after two different elections (in the same country at time $t_{1}$ and $t_{2}$ or in different countries), we will probably face different sets of parties. We saw in Section 2 that this problem can be tackled by adopting a large set $N$ of parties so that any realistic situation can be modelled by imposing $\pi_{i}=0=x_{i}$ for some $i$ 's. This raises some questions.

Is an election with 4 parties equivalent to an election with the same 4 plus 96 other parties having zero vote? If we want to analyze indices like the Rae index ${ }^{9}$, where the number of parties plays a role in the definition of the index, then our setting is not adequate, because the number of parties is some large number without any real meaning and it does not vary. Yet, for the indices characterized in this paper and for most popular indices, adding dummies does not impact the value of the disproportionality index. So, although not perfect, our setting seems fine for analyzing many interesting indices.

Is there a better setting? There is at least a more general one. We can consider a universal set $\Omega$ of parties, for instance $\Omega=\mathbb{N}$, and, for each particular election, a specific subset $N$ of $\Omega$. The index $f$ is then a function with three arguments: $N, x$ and $\pi$. We can then compare $f(N, x, \pi)$ with $f\left(N^{\prime}, x^{\prime}, \pi^{\prime}\right)$. In such a setting, it would be tempting to impose the following condition:

\footnotetext{
8 Notice that $f_{\mathrm{DD}}, f_{\mathrm{DD}}^{2}$ and $\exp \left(f_{\mathrm{DD}}\right)$ all induce the same ranking. Actually, all strictly increasing functions of a given index induce the same ranking as that index.

9 The Rae index is defined as $f_{\mathrm{DD}} / n$.
} 
$f(N, x, \pi)=f\left(N^{\prime}, x^{\prime}, \pi^{\prime}\right)$ whenever $i \notin N, N^{\prime}=N \cup\{i\}, x_{i}^{\prime}=0=\pi_{i}^{\prime}, x_{j}^{\prime}=x_{j}$ and $\pi_{j}^{\prime}=\pi_{j}$ for all $j \in N$. This amounts to saying that adding a party without votes nor seats to an election does not modify the disproportionality index. This is of course not the case for the Rae index, but it holds for most popular indices. If we impose this property, then the remaining set of indices is exactly the set we can analyze in our setting with $N$ fixed. This justifies the use of our setting. If we do not impose this property, then we can analyze a larger set of indices. We can also state some interesting properties saying, for instance, what should happen when several parties merge into a bigger one, inheriting all votes issued in favor of the original parties (as in Independence from split, [10]). This is not possible in our setting with $N$ fixed. This shows the superiority of the more general setting, but it comes at a price: a heavier formalism.

In conclusion, no setting is perfect. Thanks to the simplicity of our setting, we can use a simple notation and focus on some simple ideas underlying the Duncan and Duncan index as well as the Lijphart index.

\section{Proofs}

Lemma 1 Suppose $\pi$ is fixed. If a diversity index $f$ satisfies Homogeneity, then $f(x, \pi)=F(x / s(x))$, for some mapping $F: \Pi \rightarrow \mathbb{R}_{+}$.

Proof. Since $\pi$ is fixed, we can define a mapping $g: X \rightarrow \mathbb{R}_{+}$such that $f(x, \pi)=g(x)$. Define the mapping $F: \Pi \rightarrow \mathbb{R}_{+}$as follows. For any $p \in \Pi$, $F(p)=g(x)$ if there is $x \in X$ such that $p=x / s(x)$. The mapping $F$ is defined everywhere because $p$ has rational components and, hence, there is always $x \in X$ such that $p=x / s(x)$. The mapping $F$ is well defined. Indeed, suppose there are $x, y$ such that $p=x / s(x)$ and $p=y / s(y)$. By Homogeneity, $f(x, \pi)=f(y, \pi)$. Therefore, $F(p)=g(x)=g(y)$.

Thanks to this lemma, when $\pi$ is fixed, we can consider $f$ as a mapping defined on the $(n-1)$ dimensional simplex $\Pi$ (more precisely, the points in the simplex with rational coordinates) and we can therefore use a graphical representation when there are three categories, as in Fig. 1.

Lemma 2 Suppose $p, q$ are two elements of $\Pi$ such that $p=\alpha \pi+(1-\alpha) q$ for some $\alpha \in[0,1]$. If $f$ satisfies Homogeneity and Radial Linearity, then

$$
F\left(\frac{p+q}{2}\right)=\frac{F(p)+F(q)}{2},
$$

with $F$ defined as in Lemma 1.

Proof. Clearly, $(p+q) / 2$ belongs to $\Pi$. Since $F$ is defined everywhere, there are two parliaments $x, y$ such that $x / s(x)=p$ and $y / s(y)=q$. Hence $F(p)=f(x, \pi)$ and $F(q)=f(y, \pi)$. By Homogeneity, $F(p)=f(x, \pi)=$ $f(2 s(y) x, \pi)$ and $F(q)=f(y, \pi)=f(2 s(x) y, \pi)$. The two parliaments $s(y) x$ 


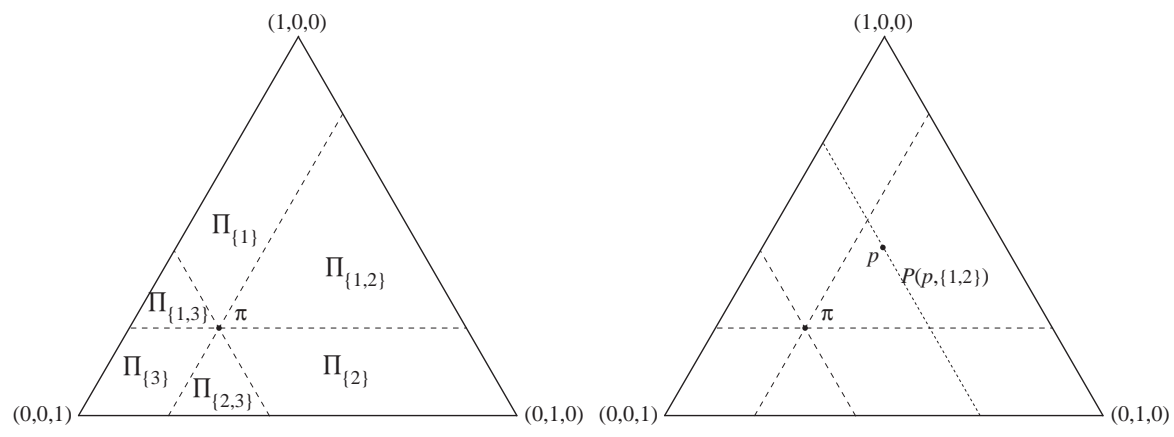

Fig. 1 Left: the domains $\Pi_{J}$. The set $\Pi$ consists of all points in the triangle with rational coordinates. Right: an affine subspace $P(p, J)$.

and $s(x) y$ have the same size, i.e., $s(x) s(y)$. Moreover, $s(y) x=\alpha s(s(y) x) \pi+$ $(1-\alpha) s(x) y$ for some $\alpha \in[0,1]$ and $(2 s(y) x+2 s(x) y) / 2 \in X$. Hence we can apply Radial Linearity and we find

$$
\frac{F(p)+F(q)}{2}=\frac{f(2 s(y) x, \pi)+f(2 s(x) y, \pi)}{2} \stackrel{\text { Rad.Lin. }}{=} f(s(y) x+s(x) y, \pi) .
$$

By definition of $F$,

$$
f(s(y) x+s(x) y, \pi)=F\left(\frac{s(y) x+s(x) y}{2 s(x) s(y)}\right)=F\left(\frac{p+q}{2}\right),
$$

from which we easily infer (2).

Let us consider a non-empty strict subset $J \subset N$ and define

$$
\Pi_{J}=\left\{p \in \Pi: p_{i} \geq \pi_{i} \forall i \in J \text { and } p_{i} \leq \pi_{i} \forall i \notin J\right\} .
$$

All these subsets are represented in Figure 1 left (in case $n=3$ ). Notice that the union of all such sets (letting $J$ vary) covers $\Pi$; formally, $\bigcup_{\emptyset \neq J \subseteq N} \Pi_{J}=\Pi$. But these sets do not partition $\Pi$ because they intersect (when some $p_{i}=\pi_{i}$ ). In particular, $\forall J \subset N, J \neq \emptyset, \pi \in \Pi_{J}$. Notice also that $\Pi_{J}$ contains only one point in some cases. More precisely, $\Pi_{J}=\{\pi\}$ iff $\pi_{i}=0$ for all $i \notin J$.

Lemma 3 Suppose $\pi$ is fixed. For every non-empty $J \subset N$, the set $\Pi_{J}$ is rational convex, i.e., $\forall p, q \in \Pi_{J}, \forall \alpha \in[0,1] \cap \mathbb{Q}, \alpha p+(1-\alpha) q \in \Pi_{J}$.

Proof. This results from the fact that $\Pi_{J}$ is the intersection of two rational convex sets: (1) the polyhedral cone with vertex $\pi$, defined by the inequalities $p_{i} \geq \pi_{i} \forall i \in J$ and $p_{i} \leq \pi_{i} \forall i \notin J$ and (2) the set $\Pi$.

Given a point $p \in \Pi$, there can be several non-empty sets $J \subset N$ such that $p \in \Pi_{J}$ (this is the case when $p_{i}=\pi_{i}$ for some $i \in N$ ). For every $p \in \Pi$, if $p \in \Pi_{J}$, we define the set $P(p, J)=\left\{q \in \Pi_{J}: \sum_{j \in J} q_{j}=\sum_{j \in J} p_{j}\right\}$. The set 
$P(p, J)$ is the intersection of the convex set $\Pi_{J}$, located in the hyperplane $\{q \in$ $\left.\mathbb{Q}^{N}: \sum_{j \in N} q_{j}=1\right\}$, with the hyperplane $\left\{q \in \mathbb{Q}^{N}: \sum_{j \in J} q_{j}=\sum_{j \in J} p_{j}\right\}$. Whenever $\Pi_{J}$ is not reduced to $\{\pi\}$, these two hyperplanes are distinct and, hence, $P(p, J)$ is located in an affine subspace of dimension $n-2$ in $\mathbb{Q}^{N}$. A set $P(p, J)$ is depicted in Fig. 1 right (with $n=3$ ). The sets $P(p, J)$ with $p$ varying in $\Pi_{J}$ form a partition of $\Pi_{J}$ because, for all $p, p^{\prime} \in \Pi_{J}$, we have $P(p, J) \neq P\left(p^{\prime}, J\right)$ iff $\sum_{j \in J} p_{j} \neq \sum_{j \in J} p_{j}^{\prime}$.

Lemma 4 Suppose $\pi$ is fixed. If $\Pi_{J} \neq\{\pi\}$, then

$$
\min _{p \in \Pi_{J}} \sum_{j \in J} p_{j}=\sum_{j \in J} \pi_{j}<\max _{p \in \Pi_{J}} \sum_{j \in J} p_{j}=1 .
$$

The maximum is attained by all $p \in \Pi_{J}$ such that $p_{j}=0$ for all $j \notin J$.

Proof. If $\Pi_{J} \neq\{\pi\}$, there is $p \in \Pi_{J}$ such that $p \neq \pi$. Hence, for some $j \in J, p_{j}>\pi_{j}$ and, consequently, $\sum_{j \in J} p_{j}>\sum_{j \in J} \pi_{j}$. Since $\pi \in \Pi_{J}$, this establishes that $\sum_{j \in J} \pi_{j}=\min _{p \in \Pi_{J}} \sum_{j \in J} p_{j}<\max _{p \in \Pi_{J}} \sum_{j \in J} p_{j}$.

Clearly, if $p$ is such that $p_{j}=0$ for all $j \notin J$, then $\sum_{j \in J} p_{j}=1$ and this sum is maximal. Such a $p$ always exists, since $J \neq N$.

Lemma 5 Suppose $\pi$ is fixed and $f$ satisfies Homogeneity and Substitution. For every non-empty $J \subset N, \forall p \in \Pi_{J}, \forall q \in P(p, J)$, we have $F(p)=F(q)$.

Proof. Consider some $p, q$ as in the statement of the lemma. By definition of $F$, there are $x, y$ such that $p=x / s(x)$ and $q=y / s(y)$. By Homogeneity, $f(x, \pi)=f(s(y) x, \pi)$ and $f(y, \pi)=f(s(x) y, \pi)$. The two parliaments $s(y) x$ and $s(x) y$ have the same size. Moreover $\sum_{j \in J} q_{j}=\sum_{j \in J} p_{j}$ and $\sum_{j \notin J} q_{j}=$ $\sum_{j \notin J} p_{j}$. We therefore have $\sum_{j \in J} s(x) y_{j}=\sum_{j \in J} s(y) x_{j}$ and $\sum_{j \notin J} s(x) y_{j}=$ $\sum_{j \notin J} s(y) x_{j}$. This means that we can 'move' from $s(x) y$ to $s(y) x$ by a series of successive exchanges between pairs of dimensions in excess or between pairs of dimension in deficit, as in the statement of Substitution. Therefore, $f(s(x) y, \pi)=f(s(y) x, \pi)$ and $F(p)=F(q)$.

Lemma 6 Suppose $\pi$ is fixed and $f$ satisfies Homogeneity and Radial Linearity. Let $J$ be a non-empty subset of $N$, and $\bar{p}$ be an element of $\Pi_{J}$ such that $\sum_{j \in J} \bar{p}_{j}=1$. Then $F$ varies linearly along the rational segment $r_{J}$ joining $\pi$ to $\bar{p}$. More precisely, for $p=\alpha \bar{p}+(1-\alpha) \pi$, with $\alpha \in[0,1] \cap \mathbb{Q}, F(p)=\alpha F(\bar{p})$. Furthermore, we have $F(p)=\gamma_{\pi}^{J} \sum_{i \in N}\left|p_{i}-\pi_{i}\right|$ for some real number $\gamma_{\pi}^{J}$.

Proof. If $\Pi_{J}=\{\pi\}$, there is nothing to prove. Otherwise, using Lemma 4, there is $\bar{p} \in \Pi_{J}$ such that $\sum_{j \in J} \bar{p}_{j}=1=\max _{p \in \Pi_{J}} \sum_{j \in J} p_{j}$. Let $p=\alpha \bar{p}+(1-$ $\alpha) \pi$, for some $\alpha \in[0,1] \cap \mathbb{Q}$. Such a point belongs to $\Pi_{J}$ since $\Pi_{J}$ is rational convex. We define the function $G:[0,1] \cap \mathbb{Q} \rightarrow \mathbb{R}_{+}$by $G(\alpha)=F(p)$. Consider another point $q=\beta \bar{p}+(1-\beta) \pi, \beta \in[0,1] \cap \mathbb{Q}$. We have $(p+q) / 2 \in \Pi_{J}$ and

$$
\frac{p+q}{2}=\frac{\alpha+\beta}{2} \bar{p}+\left(1-\frac{\alpha+\beta}{2}\right) \pi
$$


Hence (2) can be rewritten as $G\left(\frac{\alpha+\beta}{2}\right)=\frac{G(\alpha)+G(\beta)}{2}$. This is Jensen's equation and it holds for all rational $\alpha, \beta \in[0,1]$. Its unique solution is $G(\alpha)=a \alpha+b$ [1, p.43]. Hence $F(\alpha \bar{p}+(1-\alpha) \pi)=\alpha a+b$.

Letting $\alpha=0$, we obtain $F(\pi)=0=b$. Letting $\alpha=1, F(\bar{p})=a$ obtains. Hence, $F(\alpha \bar{p}+(1-\alpha) \pi)=\alpha F(\bar{p})$. This proves that $F$ varies linearly along the rational segment $r_{J}$ joining $\pi$ to $\bar{p}$.

If $p=\alpha \bar{p}+(1-\alpha) \pi$, then

$\sum_{j \in J} p_{j}=\sum_{j \in J}\left(\alpha \bar{p}_{j}+(1-\alpha) \pi_{j}\right)=\alpha \sum_{j \in J} \bar{p}_{j}+(1-\alpha) \sum_{j \in J} \pi_{j}=\sum_{j \in J} \pi_{j}+\alpha\left(1-\sum_{j \in J} \pi_{j}\right)$.

Hence $\alpha=\frac{\sum_{j \in J}\left(p_{j}-\pi_{j}\right)}{1-\sum_{j \in J} \pi_{j}}$. Since $\sum_{j \in J}\left(p_{j}-\pi_{j}\right)+\sum_{j \notin J}\left(p_{j}-\pi_{j}\right)=0$, we get $\alpha=\frac{\sum_{j \in J}\left(p_{j}-\pi_{j}\right)-\sum_{j \notin J}\left(p_{j}-\pi_{j}\right)}{2\left(1-\sum_{j \in J} \pi_{j}\right)}=\frac{\sum_{j \in J}\left(p_{j}-\pi_{j}\right)+\sum_{j \notin J}\left|p_{j}-\pi_{j}\right|}{2\left(1-\sum_{j \in J} \pi_{j}\right)}=\frac{\sum_{j \in N}\left|p_{j}-\pi_{j}\right|}{2\left(1-\sum_{j \in J} \pi_{j}\right)}$

and

$$
F(\alpha \bar{p}+(1-\alpha) \pi)=\frac{\sum_{j \in N}\left|p_{j}-\pi_{j}\right|}{2\left(1-\sum_{j \in J} \pi_{j}\right)} F(\bar{p}) .
$$

Letting $\gamma_{\pi}^{J}=\frac{F(\bar{p})}{2\left(1-\sum_{j \in J} \pi_{j}\right)}$, we obtain $F(\alpha \bar{p}+(1-\alpha) \pi)=\gamma_{\pi}^{J} \sum_{j \in N}\left|p_{j}-\pi_{j}\right|$.

Lemma $7 \forall J \subset N, J \neq \emptyset, \forall q \in \Pi_{J}, \exists p=\alpha \bar{p}+(1-\alpha) \pi$ s.t. $q \in P(p, \pi)$.

Proof. Let $\alpha=\frac{\sum_{j \in J}\left(q_{j}-\pi_{j}\right)}{\sum_{j \notin J} \pi_{j}}$. For $p=\alpha \bar{p}+(1-\alpha) \pi$, straightforward calculations show that $\sum_{j \in J} p_{j}=\sum_{j \in J} q_{j}$ and therefore $q \in P(p, \pi)$.

Lemma 8 Suppose $\pi$ is fixed and $f$ satisfies Homogeneity, Substitution and Radial Linearity. For every $J \subset N, J \neq \emptyset, \forall p \in \Pi_{J}, F(p)=\gamma_{\pi}^{J} \sum_{i \in N}\left|p_{i}-\pi_{i}\right|$ for some real number $\gamma_{\pi}^{J}$.

Proof. Fix $J$. If $\Pi_{J}=\{\pi\}$, there is nothing to prove. We suppose henceforth that $\Pi_{J} \neq\{\pi\}$. For any $p \in \Pi_{J}$, by Lemma 7 , there is $q=\alpha \bar{p}+(1-\alpha) \pi$ such that $p \in P(q, \pi)$ and, by Lemma $5, F(p)=F(q)$. By Lemma $6, F(p)=$ $F(q)=\gamma_{\pi}^{J} \sum_{i \in N}\left|q_{i}-\pi_{i}\right|$

Since $p$ and $q$ both belong to $\Pi_{J}$ and $\sum_{j \in J} q_{j}=\sum_{j \in J} p_{j}$ (see proof of Lemma 7), we have $\sum_{i \in N}\left|q_{i}-\pi_{i}\right|=\sum_{i \in N}\left|p_{i}-\pi_{i}\right|$. Therefore, $F(p)=$ $F(q)=\gamma_{\pi}^{J} \sum_{i \in N}\left|q_{i}-\pi_{i}\right|=\gamma_{\pi}^{J} \sum_{i \in N}\left|p_{i}-\pi_{i}\right|$.

Lemma 9 Suppose $\pi$ is fixed and $f$ satisfies Homogeneity, Substitution and Radial Linearity. There is some $\gamma_{\pi} \in \mathbb{R}_{++}$such that, $\forall J \subset N, J \neq \emptyset$ and for every $p \in \Pi_{J}$, we have $F(p)=\gamma_{\pi} \sum_{i \in N}\left|p_{i}-\pi_{i}\right|$. 
Proof. Let $I \subseteq N$ be such that $|I| \geq 2$ and $j \in I$. Define $J=I \backslash\{j\}$. If $\Pi_{J}=\{\pi\}$, then we can choose $\gamma_{\pi}^{J}$ equal to $\gamma_{\pi}^{I}$ without any consequence. Otherwise, there exists $p \in \Pi_{I} \cap \Pi_{J}$, with $p \neq \pi$. It is such that $p_{j}=\pi_{j}$. Because $p \in \Pi_{I} \cap \Pi_{J}$, we can write (by Lemma 8)

$$
F(p)=\gamma_{\pi}^{I} \sum_{i \in N}\left|p_{i}-\pi_{i}\right|=\gamma_{\pi}^{J} \sum_{i \in N}\left|p_{i}-\pi_{i}\right| .
$$

Since $\sum_{i \in N}\left|p_{i}-\pi_{i}\right| \neq 0$ (because $p \neq \pi$ ), we obtain $\gamma_{\pi}^{I}=\gamma_{\pi}^{J}$.

Consider now a set $I \subseteq N$ such that $|I| \leq n-2$ and $i \notin I$. Define $J=I \cup\{i\}$.

The same reasoning as above shows that $\gamma_{\pi}^{I}=\gamma_{\pi}^{J}$.

Let $I$ and $K$ be two arbitrary non-empty subsets such that $I \neq N \neq K$. We want to show that $\gamma_{\pi}^{I}=\gamma_{\pi}^{K}$. We consider two exclusive cases.

1. $I \cap K \neq \emptyset$. We rename the elements of $I \backslash K$ so that $I \backslash K=\left\{y_{1}, \ldots, y_{k}\right\}$. Using the reasoning in the first part of this proof, we find $\gamma_{\pi}^{I}=\gamma_{\pi}^{I \backslash\left\{y_{1}\right\}}=$ $\gamma_{\pi}^{I \backslash\left\{y_{1}, y_{2}\right\}}=\ldots=\gamma_{\pi}^{I \backslash(I \backslash K)}=\gamma_{\pi}^{I \cap K}$. We rename the elements of $K \backslash I$ so that $K \backslash I=\left\{z_{1}, \ldots, z_{l}\right\}$. Using the reasoning in the first part of this proof, we find $\gamma_{\pi}^{I}=\gamma_{\pi}^{I \cap K}=\gamma_{\pi}^{(I \cap K) \cup\left\{z_{1}\right\}}=\gamma_{\pi}^{(I \cap K) \cup\left\{z_{1}, z_{2}\right\}}=\ldots=\gamma_{\pi}^{(I \cap K) \cup(K \backslash I)}$. Since $(I \cap K) \cup(K \backslash I)=K$, we have proven that $\gamma_{\pi}^{I}=\gamma_{\pi}^{K}$.

2. $I \cap K=\emptyset$. We rename the elements of $I$ so that $I=\left\{y_{1}, \ldots, y_{k}\right\}$. Using the reasoning in the first part of this proof, we find $\gamma_{\pi}^{I}=\gamma_{\pi}^{I \backslash\left\{y_{1}\right\}=}$ $\gamma_{\pi}^{I \backslash\left\{y_{1}, y_{2}\right\}}=\ldots=\gamma_{\pi}^{I \backslash\left\{y_{1}, \ldots, y_{k-1}\right\}}=\gamma_{\pi}^{\left\{y_{k}\right\}}$. Notice we have not removed $y_{k}$ from $I$ to avoid considering an empty set. We will remove $y_{k}$ later.

We rename the elements of $K$ so that $K=\left\{z_{1}, \ldots, z_{l}\right\}$. Using again the reasoning in the first part of the proof of this lemma, we find $\gamma_{\pi}^{I}=\gamma_{\pi}^{\left\{y_{k}\right\}}=$ $\gamma_{\pi}^{\left\{y_{k}\right\} \cup\left\{z_{1}\right\}}=\gamma_{\pi}^{\left\{y_{k}\right\} \cup\left\{z_{1}, z_{2}\right\}}=\gamma_{\pi}^{\left\{y_{k}\right\} \cup\left\{z_{1}, \ldots, z_{l}\right\}}=\gamma^{\left\{z_{1}, \ldots, z_{l}\right\}}=\gamma_{\pi}^{K}$.

This proves that $\gamma_{\pi}^{I}=\gamma_{\pi}^{J}$ for all non-empty subsets $I, J$ of $N$. Finally, $\gamma_{\pi} \in \mathbb{R}_{++}$because we have imposed in our definition of the diversity index that $f(x, \pi) \geq 0$ for all $x \in X$ and $f(x, \pi)=0$ iff $x / s(x)=\pi$.

Proof of Theorem 1. Necessity has already been proved. We first assume $\pi$ is fixed. According to Lemma 9 and to the definition of $F$ and $g$ (see Lemma 1), we can write $f(x, \pi)=g(x)=\gamma_{\pi} \sum_{i \in N}\left|x_{i} / s(x)-\pi_{i}\right|$.

Suppose now $\pi$ is allowed to vary. By the first part of Theorem 1, we have $f(x, \pi)=\gamma_{\pi} \sum_{i \in N}\left|\frac{x_{i}}{s(x)}-\pi_{i}\right|$, for every $\pi \in \Pi$. Let $\pi^{*} \in \Pi$ be a target such that $\pi_{i}^{*}>0$ for all $i \in N$. We will show that, for any $\pi \in \Pi$, we have $\gamma_{\pi}=\gamma_{\pi^{*}}$.

Let $\lambda$ be a natural number such that $\lambda \pi, \lambda \pi^{*} \in \mathbb{N}^{N}$ and $\lambda \pi_{j}, \lambda \pi_{j}^{*} \geq 1$ for some $j \in N$. By Invariance,

$$
f\left(\lambda \pi+\mathbf{1}^{i}-\mathbf{1}^{j}, \pi\right)=2 \gamma_{\pi} / \lambda=f\left(\lambda \pi^{*}+\mathbf{1}^{i}-\mathbf{1}^{j}, \pi^{*}\right)=2 \gamma_{\pi^{*}} / \lambda .
$$

Hence $\gamma_{\pi}=\gamma_{\pi^{*}}$. This proves that $\gamma_{\pi}$ does actually not depend on $\pi$.

For the logical independence of the properties in Theorem 1, we provide four examples violating one and only one property. 
- Invariance: $f(x, \pi)=\gamma_{\pi} \sum_{i \in N}\left|\frac{x_{i}}{s(x)}-\pi_{i}\right|$, with $\gamma_{\pi}$ non-constant and positive. For instance, we could choose $\gamma_{\pi}$ in such a way that $f$ be normalized between 0 and 1, i.e., such that $\max _{x \in X} f(x, \pi)=1$.

- Homogeneity: $f(x, \pi)=\sum_{i \in N}\left|x_{i}-s(x) \pi_{i}\right|$. This index is similar to $f_{\mathrm{DD}}$ but is based on deviations between absolute frequencies instead of deviations between relative frequencies.

- Substitution (when $n \geq 3): f(x, \pi)=f_{\mathrm{L}}(x, \pi)$.

- Radial Linearity: $f(x, \pi)=\left(\sum_{i \in N}\left|\frac{x_{i}}{s(x)}-\pi_{i}\right|\right)^{2}$.

Proof of Proposition 1. Case $n=2$. Suppose $x \in \Pi_{J}$, with $J \subset N$ and $J \neq \emptyset$. We assume w.l.o.g. $J=\{1\}$. Then $\left|\frac{x_{1}}{s(x)}-\pi_{1}\right|=\left|\frac{x_{2}}{s(x)}-\pi_{2}\right|=$ $\max \left(\left|\frac{x_{1}}{s(x)}-\pi_{1}\right|,\left|\frac{x_{2}}{s(x)}-\pi_{2}\right|\right)$. Hence, $\sum_{i \in N}\left|\frac{x_{i}}{s(x)}-\pi_{i}\right|=2 \max _{i \in N}\left|\frac{x_{i}}{s(x)}-\pi_{i}\right|$.

Case $n=3$. Suppose $x \in \Pi_{J}$, with $J \subset N$ and $J$ non-empty. Two cases can arise: (1) $J=\{i\}$ or (2) $J=\{i, j\}$. In case (1), we assume w.l.o.g. $J=\{1\}$. Then $\left|\frac{x_{1}}{s(x)}-\pi_{1}\right|=\left|\frac{x_{2}}{s(x)}-\pi_{2}\right|+\left|\frac{x_{3}}{s(x)}-\pi_{3}\right|$ and $\left|\frac{x_{1}}{s(x)}-\pi_{1}\right| \geq$ $\max \left(\left|\frac{x_{2}}{s(x)}-\pi_{2}\right|,\left|\frac{x_{3}}{s(x)}-\pi_{3}\right|\right)$. Hence,

$$
\begin{aligned}
\sum_{i \in N}\left|\frac{x_{i}}{s(x)}-\pi_{i}\right| & =\left|\frac{x_{1}}{s(x)}-\pi_{1}\right|+\left|\frac{x_{2}}{s(x)}-\pi_{2}\right|+\left|\frac{x_{3}}{s(x)}-\pi_{3}\right| \\
& =2\left|\frac{x_{1}}{s(x)}-\pi_{1}\right|=2 \max _{i \in N}\left|\frac{x_{i}}{s(x)}-\pi_{i}\right| .
\end{aligned}
$$

In case (2), the reasoning is similar.

Lemma 10 (necessity of some conditions for $f_{L}$ ). Index $f_{L}$ satisfies Homogeneity, Radial Linearity, Substitution* and Invariance.

Proof. We already proved that Substitution* is necessary and Homogeneity obviously holds. Let us show the necessity of Radial Linearity. We have

$f_{\mathrm{L}}\left(\frac{x+y}{2}, \pi\right)=\max _{i \in N}\left|\pi_{i}-\frac{x_{i}+y_{i}}{2 s(x)}\right|=\frac{1}{2} \max _{i \in N}\left|2 \pi_{i}-\frac{x_{i}+y_{i}}{s(x)}\right|=\frac{1}{2} \max _{i \in N}\left|\pi_{i}-\frac{x_{i}}{s(x)}+\pi_{i}-\frac{y_{i}}{s(y)}\right|$.

Using $x=\alpha s(x) \pi+(1-\alpha) y$ and the fact that $\pi_{i}-x_{i} / s(x) \geq 0$ iff $\pi_{i}-y_{i} / s(y) \geq$ 0 , we have $f_{\mathrm{L}}\left(\frac{x+y}{2}, \pi\right)$

$$
\begin{aligned}
& =\frac{1}{2} \max _{i \in N}\left|\pi_{i}-\frac{x_{i}}{s(x)}+\pi_{i}-\frac{y_{i}}{s(y)}\right|=\frac{1}{2} \max _{i \in N}\left(\left|\pi_{i}-\frac{x_{i}}{s(x)}\right|+\left|\pi_{i}-\frac{y_{i}}{s(y)}\right|\right) \\
& =\frac{1}{2}\left(\max _{i \in N}\left|\pi_{i}-\frac{x_{i}}{s(x)}\right|+\max _{i \in N}\left|\pi_{i}-\frac{y_{i}}{s(y)}\right|\right)=\frac{f_{\mathrm{L}}(x, \pi)+f_{\mathrm{L}}(y, \pi)}{2} .
\end{aligned}
$$

Necessity of Invariance: $f_{\mathrm{L}}\left(\lambda \pi+\mathbf{1}^{i}-\mathbf{1}^{j}, \pi\right)=\left|\frac{\lambda \pi_{i}+1}{\lambda}-\pi_{i}\right|=1 / \lambda$. In the same way, $f_{\mathrm{L}}\left(\lambda \pi^{\prime}+\mathbf{1}^{i}-\mathbf{1}^{j}, \pi^{\prime}\right)=1 / \lambda$. This proves that $f_{\mathrm{L}}\left(\lambda \pi+\mathbf{1}^{i}-\mathbf{1}^{j}, \pi\right)=$ 
$f_{\mathrm{L}}\left(\lambda \pi^{\prime}+\mathbf{1}^{i}-\mathbf{1}^{j}, \pi^{\prime}\right)$.

For every $i \in N, \pi$ being fixed, define $\Pi_{i}^{+}=\left\{p \in \Pi: p_{i}-\pi_{i} \geq\right.$ $\left.\left|p_{j}-\pi_{j}\right| \forall j \in N \backslash\{i\}\right\}$ and $\Pi_{i}^{-}=\left\{p \in \Pi: \pi_{i}-p_{i} \geq\left|p_{j}-\pi_{j}\right| \forall j \in N \backslash\{i\}\right\}$. If $n=2$ or 3 , then, $\forall i \in N, \Pi_{i}^{+}=\Pi_{\{i\}}$ and $\Pi_{i}^{-}=\Pi_{N \backslash\{i\}}\left(\Pi_{\{i\}}\right.$ has been defined just after the proof of Lemma 2$)$. But these identities are no longer true when $n>3$. Notice that $\bigcup_{i \in N}\left(\Pi_{i}^{+} \cup \Pi_{i}^{-}\right)=\Pi$. These sets do not partition $\Pi$ because they intersect. In particular, $\forall i \in N$, the point $\pi$ belongs to $\Pi_{i}^{+}$ and $\Pi_{i}^{-}$. Notice also that $\Pi_{i}^{+}$and $\Pi_{i}^{-}$contain only one point in some cases. More precisely, $\Pi_{i}^{+}=\{\pi\}$ if $\pi_{i}=1$. Similarly, $\Pi_{i}^{-}=\{\pi\}$ if $\pi_{i}=0$.

Lemma 11 Suppose $\pi$ is fixed. For $i \in N$, the set $\Pi_{i}^{+}$is rational convex, i.e., $\forall p, q \in \Pi_{i}^{+}, \forall \alpha \in[0,1] \cap \mathbb{Q}, \alpha p+(1-\alpha) q \in \Pi_{i}^{+} . \Pi_{i}^{-}$is also rational convex.

Proof. This results from the fact that $\Pi_{i}^{+}$is the intersection of $n$ rational convex sets: (1) $n-1$ sets corresponding to the inequalities $p_{i}-\pi_{i} \geq$ $\left|p_{j}-\pi_{j}\right| \forall j \in N \backslash\{i\}$ and (2) the set $\Pi$. The proof for $\Pi_{i}^{-}$is similar

Given a point $p \in \Pi$, there can be several $i \in N$ such that $p \in \Pi_{i}^{+}$ or $p \in \Pi_{i}^{-}$. For every $p \in \Pi$, if $p \in \Pi_{i}^{+}$, we define the set $P^{+}(p, i)=$ $\left\{q \in \Pi_{i}^{+}: q_{i}=p_{i}\right\}$ and, if $p \in \Pi_{i}^{-}$, we define the set $P^{-}(p, i)=\left\{q \in \Pi_{i}^{-}: q_{i}=p_{i}\right\}$. Notice that $P^{+}(p, i)$ and $P^{-}(p, i)$ are included in an affine subspace of dimension $n-2$ containing $p$. This affine subspace is the intersection of the hyperplane $\sum_{i \in N} p_{i}=1$ with the hyperplane defined by $q_{i}=p_{i}$.

Lemma 12 Suppose $\pi$ is fixed and the diversity index $f$ satisfies Homogeneity and Substitution* * For every $i \in N$, for every $p \in \Pi_{i}^{+}\left(\right.$resp. $\left.\Pi_{i}^{-}\right)$,

1. $\forall q \in P^{+}(p, i)$ (resp. $\left.P^{-}(p, i)\right)$, we have $F(p)=F(q)$, with $F$ as in Lemma 1 ;

2. $F(p)$ depends only on $p_{i}$.

Proof. We prove it only for $p \in \Pi_{i}^{+}$and $q \in P^{+}(p, i)$.

(1) Consider some $p, q$ as in the statement of the lemma. By definition of $F$, there are $x, y$ such that $p=x / s(x)$ and $q=y / s(y)$. By Homogeneity, $f(x, \pi)=$ $f(s(y) x, \pi)$ and $f(y, \pi)=f(s(x) y, \pi)$. The two parliaments $s(y) x$ and $s(x) y$ have the same size. Moreover $q_{i}=p_{i}$. We therefore have $s(x) y_{i}=s(y) x_{i}$. This means that we can 'move' from $s(x) y$ to $s(y) x$ by a series of successive exchanges between pairs of dimensions different from $i$, as in the statement of Substitution*. Therefore, $f(s(x) y, \pi)=f(s(y) x, \pi)$ and $F(p)=F(q)$.

(2) For every $q \in P^{+}(p, i), F(q)=F(p)$. Besides, $q \in P^{+}(p, i)$ iff $q_{i}=p_{i}$. Therefore, $F(q)$ only depends on $q_{i}$ and $F(p)$ only depends on $p_{i}$.

Lemma 13 Suppose $\pi$ is fixed. Define the line segment $r^{i}$ (resp. $s^{i}$ ) with endpoints $\pi$ and $\mathbf{1}^{i}$ (resp. $\pi$ and $\left(\pi-\left(\pi_{i} \mathbf{1}^{i}\right)\right) /\left(1-\pi_{i}\right)$ ). For every $p \in \Pi_{i}^{+}$, the set $P^{+}(p, i)$ intersects $r^{i}$. For every $p \in \Pi_{i}^{-}$, the set $P^{-}(p, i)$ intersects $s^{i}$. 
Proof. By construction, $r^{i} \in \Pi_{i}^{+}$and $s^{i} \in \Pi_{i}^{-}$. For every $p \in \Pi_{i}^{+}$, we have $\pi_{i} \leq p_{i} \leq 1$. By construction, there is $q \in r^{i}$ such that $q_{i}=p_{i}$. Hence $q \in P^{+}(p, i)$. For every $p \in \Pi_{i}^{-}$, we have $0 \leq p_{i} \leq \pi_{i}$. By construction, there is $q \in s^{i}$ such that $q_{i}=p_{i}$. Hence $q \in P^{-}(p, i)$.

Lemma 14 Suppose $\pi$ is fixed and $f$ satisfies Homogeneity and Radial Linearity. For each $i \in N, F$ varies linearly along $r^{i}$ in $\Pi_{i}^{+}$(resp. $s^{i}$ in $\Pi_{i}^{-}$).

Proof. We prove it only for $\Pi_{i}^{+}$. If $\Pi_{i}^{+}=\{\pi\}$, there is nothing to prove. We suppose henceforth that $\Pi_{i}^{+} \neq\{\pi\}$. Every point of $r^{i}$ can be written as $\alpha \pi+(1-\alpha) \bar{p}$, with $\alpha \in[0,1] \cap \mathbb{Q}$. Define the mapping $G:[0,1] \cap \mathbb{Q} \rightarrow \mathbb{R}_{+}$ by $G(\alpha)=F(p)$ if $p=\alpha \pi+(1-\alpha) \bar{p}$. $G$ is clearly well-defined. Consider any two points $p, q \in r^{i}$, the position of which on $r^{i}$ is characterized by $\alpha$ and $\beta$ respectively. Clearly, $(p+q) / 2 \in r^{i}$ and $\frac{p+q}{2}=\frac{\alpha+\beta}{2} \pi+\left(1-\frac{\alpha+\beta}{2}\right) \bar{p}$ with $(\alpha+\beta) / 2 \in[0,1] \cap \mathbb{Q}$. Hence $(2)$ can be written as $G\left(\frac{\alpha+\beta}{2}\right)=\frac{G(\alpha)+G(\beta)}{2}$. This is Jensen's equation and it holds for all $\alpha, \beta \in[0,1] \cap \mathbb{Q}$. Its unique solution is $G(\alpha)=a \alpha+b[1, \mathrm{p} .43]$. Hence, $F$ varies linearly along the line segment $r^{i}$.

Lemma 15 Suppose $\pi$ is fixed and the diversity index $f$ satisfies Homogeneity, Substitution* and Radial Linearity. For every $i \in N$, for every $p \in \Pi_{i}^{+}$, $F(p)=\gamma_{i, \pi}^{+} \max _{i \in N}\left|p_{i}-\pi_{i}\right|$ for some positive real number $\gamma_{i, \pi}^{+}$. Similarly, for every $i \in N$, for every $p \in \Pi_{i}^{-}, F(p)=\gamma_{i, \pi}^{-} \max _{i \in N}\left|p_{i}-\pi_{i}\right|$ for some positive real number $\gamma_{i, \pi}^{-}$.

Proof. We prove it only for $\Pi_{i}^{+}$. Fix $i$. If $\Pi_{i}^{+}=\{\pi\}$, there is nothing to prove. We suppose henceforth that $\Pi_{i}^{+} \neq\{\pi\}$. We know from Lemma 12 that $F$ is constant in each set $P^{+}(p, i)$, for all $p \in \Pi_{i}^{+}$, and depends only on $p_{i}$. By Lemma 13, we know that each set $P^{+}(p, i)$ intersects the line segment $r^{i}$. Finally, Lemma 14 tells us that $F$ varies linearly along $r^{i}$.

In conclusion, $F(p)=\gamma_{i, \pi}^{+} p_{i}+\delta_{i}^{+}$for some real numbers $\gamma_{i, \pi}^{+}, \delta_{i}^{+}$. Notice that these real numbers are indexed by $i$ because they can be different in every set $\Pi_{i}^{+}$. The definition of a diversity index imposes that $F(\pi)=\gamma_{i, \pi}^{+} \pi_{i}+\delta_{i}^{+}=0$. Hence, $\delta_{i}^{+}=-\gamma_{i, \pi}^{+} \pi_{i}$ and $F(p)=\gamma_{i, \pi}^{+}\left(p_{i}-\pi_{i}\right)=\gamma_{i, \pi}^{+} \max _{j \in N}\left|p_{j}-\pi_{j}\right|$.

Lemma 16 Suppose $n \geq 3, \pi$ is fixed and the diversity index $f$ satisfies Homogeneity, Substitution * and Radial Linearity. There exists $\gamma_{\pi} \in \mathbb{R}_{++}$such that, for every $i \in N$ and for every $p \in \Pi_{i}^{+}, F(p)=\gamma_{\pi} \max _{i \in N}\left|p_{i}-\pi_{i}\right|$.

Proof. Choose some $i \in N$ with $\Pi_{i}^{+} \neq\{\pi\}$. Choose some other $j \in N$. If $\Pi_{j}^{-}=\{\pi\}$, then we can choose $\gamma_{j, \pi}^{-}$equal to $\gamma_{i, \pi}^{+}$without any consequence. Otherwise, define $p$ by $p_{i}=\pi_{i}+\epsilon, p_{j}=\pi_{j}-\epsilon$ and $p_{k}=\pi_{k}$ for every $k \neq i, j$. If we choose $\epsilon$ small enough, we are sure that $p \in \Pi_{i}^{+} \cap \Pi_{j}^{-}$, with $p \neq \pi$. Because $p \in \Pi_{i}^{+} \cap \Pi_{j}^{-}$, we have (by Lemma 15) $F(p)=\gamma_{i, \pi}^{+} \max _{i \in N}\left|p_{i}-\pi_{i}\right|=$ 
$\gamma_{j, \pi}^{-} \max _{i \in N}\left|p_{i}-\pi_{i}\right|$. Since $\max _{i \in N}\left|p_{i}-\pi_{i}\right| \neq 0$ (because $p \neq \pi$ ), we obtain $\gamma_{i, \pi}^{+}=\gamma_{j, \pi}^{-}$. We can repeat the same reasoning and we then find for instance $\gamma_{i, \pi}^{+}=\gamma_{j, \pi}^{-}=\gamma_{k, \pi}^{+}=\gamma_{i, \pi}^{-}=\ldots$ This proves that $\gamma_{i, \pi}^{+}=\gamma_{j, \pi}^{+}=\gamma_{i, \pi}^{-}=\gamma_{j, \pi}^{-}$for all $i, j \in N$. Finally, $\gamma_{\pi} \in \mathbb{R}_{++}$because, by definition of the diversity index, $f(x, \pi) \geq 0$ for all $x \in X$ and $f(x, \pi)=0$ iff $x / s(x)=\pi$.

Proof of Theorem 2. Necessity has been proved in Lemma 10. If $n=$ 2, Substitution and Substitution* are vacuous and, hence, the properties of theorems 2 and 1 are equivalent. We therefore know that $f=\gamma_{\pi} f_{\mathrm{DD}}$ for some $\gamma_{\pi}$. By Proposition 1 , we also know that $f_{\mathrm{DD}}=2 f_{\mathrm{L}}$. In conclusion, $f=2 \gamma_{\pi} f_{\mathrm{L}}$.

We now assume $\pi$ is fixed and $n \geq 3$. Thanks to Lemma 16 and the definition of $F$ and $g$ (Lemma 1 ), we find $f(x, \pi)=g(x)=\gamma_{\pi} \max _{i \in N}\left|x_{i} / s(x)-\pi_{i}\right|$.

We turn to the case where $\pi$ is allowed to vary. By Theorem 2 , we know that, for every $\pi \in \Pi, f(x, \pi)=\gamma_{\pi} \max _{i \in N}\left|\frac{x_{i}}{s(x)}-\pi_{i}\right|$. Let $\pi^{*} \in \Pi$ be a target such that $\pi_{i}^{*}>0$ for all $i \in N$. We will show that, $\forall \pi \in \Pi$, we have $\gamma_{\pi}=\gamma_{\pi^{*}}$. Let $\lambda$ be a natural number such that $\lambda \pi, \lambda \pi^{*} \in \mathbb{N}^{N}$ and $\lambda \pi_{j}, \lambda \pi_{j}^{*} \geq 1$ for some $j \in N$. By Invariance, $f\left(\lambda \pi+\mathbf{1}^{i}-\mathbf{1}^{j}, \pi\right)=\gamma_{\pi} / \lambda=f\left(\lambda \pi^{*}+\mathbf{1}^{i}-\mathbf{1}^{j}, \pi^{*}\right)=\gamma_{\pi^{*}} / \lambda$. Hence $\gamma_{\pi}=\gamma_{\pi^{*}}$. This proves that $\gamma_{\pi}$ does actually not depend on $\pi$ and that $f$ can be written as in the statement of Theorem 2 .

For the logical independence of the properties in Theorem 2, we provide four examples violating one and only one property.

- Invariance: $f(x, \pi)=\gamma_{\pi} \max _{i \in N}\left|\frac{x_{i}}{s(x)}-\pi_{i}\right|$, with $\gamma_{\pi}>0$ and non-constant.

- Homogeneity: $f(x, \pi)=\max _{i \in N}\left|x_{i}-s(x) \pi_{i}\right|$.

- Substitution*: $f(x, \pi)=f_{\mathrm{DD}}(x, \pi)$.

- Radial Linearity: $f(x, \pi)=\left(\max _{i \in N}\left|\frac{x_{i}}{s(x)}-\pi_{i}\right|\right)^{2}$.

\section{References}

1. Aczél J (1966) Lectures on functional equations and their applications. Academic Press

2. Balinski M, Young HP (1975) The quota method of apportionment. The American Mathematical Monthly 82(7):701-730

3. Bell DE, Raiffa H, Tversky A (1988) Decision Making: Descriptive, Normative, and Prescriptive Interactions. Cambridge University Press

4. Bolun I (2012) Comparison of indices of disproportionality in PR systems. Computer Science Journal of Moldova 20(2):246-271

5. Bouyssou D, Marchant T (2007) An axiomatic approach to noncompensatory sorting methods in MCDM, II: More than two categories. European Journal of Operational Research 178:246-276

6. Bouyssou D, Pirlot M (2004) Following the traces: An introduction to conjoint measurement without transitivity and additivity. European Journal of Operational Research 163:287-337 
7. Cha SH (2007) Comprehensive survey on distance/similarity measures between probability density functions. International Journal of Mathematical Models and Methods in Applied Sciences 4(1):300-307

8. Duncan OD, Duncan B (1955) A methodological analysis of segregation indexes. American Sociological Review 20(2):210-217

9. Fields GS, Ok EA (1996) The meaning and measurement of income mobility. Journal of Economic Theory 71(2):349-377

10. Karpov A (2008) Measurement of disproportionality in proportional representation. Mathematical and Computer Modelling 48:1421-1438

11. Koppel M, Diskin A (2009) Measuring disproportionality, volatility and malapportionment: axiomatization and solutions. Social Choice and Welfare 33:281-286

12. Lijphart A (1994) Electoral Systems and Party Systems. Oxford University Press, Oxford

13. Loosemore J, Hanby V (1971) The theoretical limits of maximum distortion: Some analytical expressions for electoral systems. British Journal of Political Science 1(467-77)

14. Luce RD, Krantz DH, Suppes P, Tversky A (1990) Foundations of measurement, vol. 3: Representation, axiomatisation and invariance. Academic Press, New York

15. Nagel SS (1984) Public Policy: Goals, Measurement, and Methods. St. Martin's Press, New York

16. Nurmi H (2014) Some remarks on the concept of proportionality. Annals of Operations Research 215:231-244

17. Rae DW (1967) The political consequences of electoral laws. Yale University Press, New Haven

18. Taagepera R, Grofman B (2003) Mapping the indices of seats-votes disproportionality and inter-election volatility. Party Politics 9:659-677

19. Thomson W (2001) On the axiomatic method and its recent applications to game theory and resource allocation. Social Choice and Welfare 18:327386 